\title{
Influence of Cytochrome P450 2D6 Polymorphisms on the Efficacy of Oral Propranolol in Treating Infantile Hemangioma
}

\author{
Lidan Wang $\mathbb{D}^{1},{ }^{1}$ Kai Zheng, ${ }^{2}$ Xinlin $\mathrm{Li}^{3}{ }^{3}$ Yang Wang, ${ }^{3}$ and Qiong Xu $\mathbb{D}^{3}$ \\ ${ }^{1}$ Department of Radiology, Wuhan Children's Hospital, Tongji Medical College, \\ Huazhong University of Science \& Technology, China \\ ${ }^{2}$ Department of General Surgery, Wuhan Children's Hospital, Tongji Medical College, \\ Huazhong University of Science \& Technology, China \\ ${ }^{3}$ Department of Clinical Pharmacology, Wuhan Children's Hospital, Tongji Medical College, \\ Huazhong University of Science \& Technology, China
}

Correspondence should be addressed to Qiong Xu; polarisyxt@hotmail.com

Received 28 November 2019; Revised 21 February 2020; Accepted 26 February 2020; Published 7 March 2020

Academic Editor: Gilbert Lefèvre

Copyright (c) 2020 Lidan Wang et al. This is an open access article distributed under the Creative Commons Attribution License, which permits unrestricted use, distribution, and reproduction in any medium, provided the original work is properly cited.

\begin{abstract}
Objective. The aim of this study is to evaluate the association of genetic polymorphisms in Cytochrome P450 2D6(CYP2D6) and the change in VEGF levels with the response to propranolol in patients with Infantile hemangiomas (IH). Methods. IH patients who underwent over six months of propranolol therapy and received oral propranolol only were enrolled. The target dose of propranolol was $1 \mathrm{mg} \mathrm{kg}^{-1} \mathrm{day}^{-1}$. Deoxyribonucleic acid was obtained from venous blood leukocytes. Genotypes of CYP2D6 (rs1065852 and rs1135840) were tested by polymerase chain reaction (PCR) and by sequencing the products. Baseline serum VEGF and serum VEGF one month after treatment were measured. The clinical responses after six months of treatment were evaluated. Genotypes of CYP2D6 (rs1065852 and rs1135840) and VEGF levels were compared between good responders and poor-to-moderate responders. Results. 72 patients were enrolled in the study. Patients with CYP2D6 (rs1135840) G/G homozygote had the highest response rate to propranolol. No significant association was found between the response rates and CYP2D6 (rs1065852) polymorphism. No significant differences were found in baseline serum VEGF, serum VEGF one month after treatment, and VEGF ratio between good responders and poor-to-moderate responders. Conclusion. The response to propranolol treatment in IH patients was associated with the gene polymorphism of CYP2D6 (rs1135840). A low-dose propranolol regimen was effective and safe in young infants with IH. The change of serum VEGF levels after one month's treatment could not be used to predict the response rate to propranolol.
\end{abstract}

\section{Introduction}

Infantile hemangiomas ( $\mathrm{IH}$ ) occur in $3 \%$ to $10 \%$ of infants [1]. Not all IH need treatment as IH have proliferative phase and spontaneous regression phase, and most $\mathrm{IH}$ can regress spontaneously. However, IH that can cause disfigurement and serious complications need treatment [2].

Oral propranolol is widely used in treating complicated IH [3]. However, the efficacy of propranolol on IH varies. Some patients failed propranolol treatment $[4,5]$. Though the reported propranolol-resistant IH were rare, it is troublesome for patients who fail propranolol treatment as they often need to receive other individualized treatments such as surgical therapy [6], laser therapy [7], or injection of bleomycin [8].

During the growth phase, the growth speed of $\mathrm{IH}$ is different. Without effective intervention, the size of most $\mathrm{IH}$ (80\%) will double, in $5 \%$ of patients, the size will triple, and in less than $5 \%$, the size will dramatically extend [9]. When the effects of propranolol are evaluated after six months of therapy as in most studies, the lesions would have already become larger in the poor responders. If the alternative treatments are administered to the poor responders with larger lesions, it would be more painful for them because the wound of the alternative treatments would also be larger. Therefore, clinical or biological markers that can predict poor response 
to propranolol therapy will be useful for prognosis and enable early individualized treatments in the poor responders.

However, researches about markers of the poor response to propranolol are limited. A study tried to find the correlation between heart rate reduction after propranolol administration and the clinical outcome of propranolol therapy for IH. But no relationship was proved after six months of propranolol therapy [10].

It was reported that CYP2D6 was of great importance in propranolol metabolism [11]. Studies showed different CYP2D6 activities derived from the Cytochrome P450 2D6 (CYP2D6) polymorphism $[12,13]$. Poor metabolizers of the CYP2D6 C/T188 genotypes had less activity, and therefore, the plasma concentrations of propranolol were higher in patients with poor metabolizers than in those with extensive metabolizers [14]. It is possible that the CYP2D6 genetic polymorphisms may affect the outcome of propranolol therapy for IH. However, studies about the association between CYP2D6 genetic polymorphisms and the clinical effects of propranolol on IH are rare.

Increased vascular endothelial growth factor (VEGF) level is considered as one of the mechanisms of IH. Serum VEGF levels increased in the growth phase of IH and significantly decreased in the involuting phase [15]. It was shown that propranolol inhibited VEGF expression [16]. A study showed that the decreases of serum VEGF concentrations were more obvious after one month of propranolol therapy than those after three months of therapy [17]. Another study showed that the biggest change measured in Hemangioma Activity Score (HAS) occurred after one month of propranolol treatment [18]. Maybe the changes in VEGF after one month of treatment would be correlated with the response rate to propranolol and could be used to predict the response to propranolol.

In this study, we conducted a prospective study in children with IH to investigate the relationship between CYP2D6 (rs1065852 and rs1135840) gene polymorphisms and the efficacy of propranolol on children with $\mathrm{IH}$. The predictive value of the change in serum VEGF was also investigated.

\section{Materials and Methods}

2.1. Patients. Between June 2017 and January 2019, children with newly diagnosed $\mathrm{IH}$ who underwent over six months of propranolol therapy and received oral propranolol only were enrolled in the study. The study was approved by the Research Ethics Board of Wu Han Children's Hospital and was conducted in conformity with the ethical principles of the Declaration of Helsinki. Consent of the patients' legal guardians was obtained.

2.2. Treatment. Before oral propranolol treatment, echocardiography and electrocardiography were performed. Patients with mixed or deep IH underwent magnetic resonance imaging (MRI). Parents or guardians were informed of signs of adverse drug reactions (including hypoglycemia, hypotension, bradycardia, and bronchospasm) and precautionary measures.
Propranolol tablet (10 mg a tablet) was administered once a day orally to patients in the morning immediately before feeding. The initial dose was $0.5 \mathrm{mg} \mathrm{kg}^{-1} \mathrm{day}^{-1}$, and the dose increased to the target dose $\left(1 \mathrm{mg} \mathrm{kg}^{-1} \mathrm{day}^{-1}\right)$ on the eighth day.

Monthly visits were conducted after reaching the target dose. The dose of propranolol was altered based on weight change at each visit.

Clinical data were recorded at each visit by a clinician including age, gender, body weight, height, serum creatinine $(\mathrm{Scr})$, alanine aminotransferase (ALT), blood glucose, heart rate, IH classification, number of lesions, location, and propranolol dosage. The size of the hemangioma lesion was measured and documented at each visit. Digital photographs were taken. Changes in color and lesion size and hemangioma development (improvement, stabilization, or aggravation) were assessed and compared with previous visits. Patients with mixed or deep IH underwent MRI after six months of treatment. The clinical responses after six months of treatment were evaluated. Treatments with complete or approximately complete resolutions of the lesions were considered successful ones. The approximately complete resolution was defined as a slight degree of IH lesion [19]. Patients with a successful treatment were categorized as good responders. The rest of the patients were categorized as poor-to-moderate responders.

As superficial IH occur earlier and involution of them begin sooner than deeper ones [7], the differences of the clinical IH types (superficial, deep, and mixed components) between good responders and poor-to-moderate responders were compared. As segmental hemangiomas needed longer therapy and had a poorer overall outcome [20], to exclude the influence of the longer treatment durations of segmental hemangiomas on the outcome of our study, the constituent ratios of focal, segmental, and multifocal hemangiomas were also compared between good responders and poor-tomoderate responders.

After six months of treatment, propranolol was gradually withdrawn in patients with complete resolution. Treatment continued to 12 months if the regression of the lesion was lower than $90 \%$. No patients received propranolol therapy for over 12 months.

Blood samples were drawn before and at one month after the initiation of propranolol treatment. Serum samples were obtained and stored at $-20^{\circ} \mathrm{C}$.

2.3. Determination of Serum VEGF. Serum VEGF levels were determined by Human VEGF Enzyme-Linked Immunosorbent Assay (ELISA) Kits EK183-96 (Multisciences (Lianke) Biotech, Co., Ltd., Hangzhou, China). The detection range of the ELISA Kit was 1-1000 $\mathrm{pg} \mathrm{ml}^{-1}$. All serum VEGF levels in the study were within the detection range of the Kits and were tested in accordance with the instruction of the Kit.

Baseline serum VEGF levels were the VEGF levels measured one day before the propranolol administration. Serum VEGF ratios were got by dividing the serum VEGF levels one month after the treatment by the baseline VEGF levels.

2.4. CYP2D6 SNP Genotyping. Deoxyribonucleic acid was obtained from venous blood leukocytes. Genotypes of 
TABLE 1: Patients' characteristics and serum VEGF levels.

\begin{tabular}{|c|c|c|c|c|}
\hline Characteristics & Total patients & Good responders & Poor-to-moderate responders & $p^{\#}$ \\
\hline Patient number $n(\%)$ & 72 & $54(75)$ & $18(25)$ & \\
\hline Corrected age at inclusion (days) [median (IQR)] & $65.5(48.2-93.8)$ & $70.0(47.5-100.0)$ & $53.5(46.0-88.0)$ & $0.191^{\mathrm{b}}$ \\
\hline Gender (male/female) $(n)$ & $16 / 56$ & $12 / 42$ & $4 / 14$ & $1.0^{\mathrm{C}}$ \\
\hline Body weight $(\mathrm{kg})$ [median (IQR)] & $5.5(4.8-6.4)$ & $5.5(5.0-6.5)$ & $5.5(4.5-6.1)$ & $0.399^{\mathrm{b}}$ \\
\hline Height $(\mathrm{cm})[$ mean $(\mathrm{SD})] /[$ median $(\mathrm{IQR})]$ & $57.32(4.71)$ & $57.0(54.0-61.1)$ & $56.0(53.8-58.3)$ & $0.344^{\mathrm{b}}$ \\
\hline Blood glucose $\left(\mathrm{mmol} \mathrm{L}^{-1}\right)$ [mean $\left.(\mathrm{SD})\right]$ & $4.93(0.36)$ & $4.95(0.37)$ & $4.86(0.32)$ & $0.366^{\mathrm{a}}$ \\
\hline $\operatorname{ALT}\left(\mathrm{U} \mathrm{L}^{-1}\right)[$ mean $(\mathrm{SD})]$ & $30.00(7.95)$ & $30.28(7.73)$ & $29.17(8.78)$ & $0.611^{\mathrm{a}}$ \\
\hline $\operatorname{Scr}\left(\mu \mathrm{mol} \mathrm{L}^{-1}\right)[$ mean $(\mathrm{SD})]$ & $21.00(3.50)$ & $20.84(3.42)$ & $21.50(3.80)$ & $0.491^{\mathrm{a}}$ \\
\hline Baseline heart rate (beats per minute) [mean (SD)] & $129.79(6.53)$ & $129.46(6.35)$ & $130.78(7.14)$ & $0.463^{\mathrm{a}}$ \\
\hline Clinical IH types & & & & $0.905^{\mathrm{c}}$ \\
\hline Superficial component $n(\%)$ & $39(54.2)$ & $30(55.6)$ & $9(50.0)$ & \\
\hline Deep component $n(\%)$ & $4(5.6)$ & $3(5.6)$ & $1(5.6)$ & \\
\hline Mixed component $n(\%)$ & $29(40.3)$ & $21(38.9)$ & $8(44.4)$ & \\
\hline Morphologic classification & & & & $0.517^{\mathrm{C}}$ \\
\hline Localized $n(\%)$ & $51(70.8)$ & $36(66.7)$ & $15(83.3)$ & \\
\hline Segmental $n(\%)$ & $13(18.1)$ & $11(20.4)$ & $2(11.1)$ & \\
\hline Multifocal $n(\%)$ & $8(11.1)$ & $7(13.0)$ & $1(5.6)$ & \\
\hline Baseline serum VEGF $\left(\mathrm{pg} \mathrm{m}^{-1}\right)$ [median (IQR)] & $202.69(149.72-251.54)$ & $201.35(156.16-240.73)$ & $213.22(114.75-255.08)$ & $0.682^{\mathrm{b}}$ \\
\hline $\begin{array}{l}\text { Serum VEGF } 1 \text { month after treatment }\left(\mathrm{pg} \mathrm{ml}^{-1}\right) \\
{[\text { median }(\mathrm{IQR})]}\end{array}$ & $168.07(101.91-222.31)$ & $169.35(105.37-219.71)$ & $154.59(88.30-253.27)$ & $0.886^{\mathrm{b}}$ \\
\hline Serum VEGF ratio [median (IQR)] & $0.82(0.65-1.04)$ & $0.81(0.62-1.04)$ & $0.84(0.71-1.04)$ & $0.603^{b}$ \\
\hline
\end{tabular}

CYP2D6 (rs1065852 and rs1135840) were tested by polymerase chain reaction (PCR) and by sequencing the products. The PCR primers were designed and synthesized by Shanghai Thermo Fisher Scientific (China) Co., Ltd. PCR was performed by Bio-rad S1000 Thermal Cycler. Genotyping was performed by sanger sequencing.

2.5. Statistical Analysis. Statistical analysis was performed using the Statistical Package for the Social Sciences (SPSS version 21.0, SPSS Inc., Chicago, IL, USA). The normality of the distributions of continuous variables was checked by Kolmogorov-Smirnov test. Normally distributed continuous variables were compared using a Student's $t$ test. Nonnormally distributed continuous variables data were compared with a Mann-Whitney $U$ test. Categorical data were compared using a chi-square test. Variables with a two-tailed $p$ value $<0.05$ were considered statistically significant.

\section{Results}

72 patients were enrolled. The patients' demographic characteristics and serum VEGF levels are presented in Table 1. 56 patients were female $(77.8 \%)$, and 16 were male $(22.2 \%)$. The median age of the patients at propranolol initiation was 65.5 days (range, 33-274 days). 52 patients were younger than 3 months old at propranolol initiation.

IH can be morphologically categorized into 4 groups: localized, segmental, indeterminate, and multifocal hemangi- omas [9]. However, in our study, no patients presented indeterminate hemangiomas. 51 patients $(70.8 \%)$ had localized lesions, 13 patients (18.1\%) had segmental lesions, while 8 patients (11.1\%)had multifocal lesions. The lesions were located on facial areas in 46 patients $(63.9 \%)$ and on nonfacial areas in $26(36.1 \%) .54$ patients had good responses to propranolol after six months of therapy. Typical cases are shown in Figures 1-3. There were no significant differences between good responders and poor-to-moderate responders in gestationally corrected age at inclusion, gender, body weight, height, ALT, Scr, baseline heart rate, baseline blood pressure, morphologic classification, and the clinical IH types (superficial, deep, and mixed components).

Baseline serum VEGF, serum VEGF one month after treatment, and VEGF ratio were compared between good responders and poor-to-moderate responders, but no significant differences were found.

3.1. Patients' Genotype Distribution. The allelic frequencies of CYP2D6 (rs1065852 and rs1135840) were consistent with the Hardy-Weinberg equilibrium.

The genotype of rs1065852 and rs1135840 and their associations with the treatment responses were shown in Table 2, respectively.

After six months of treatment, no significant association was found between the response rates and CYP2D6 (rs1065852) polymorphism. Patients with the G/G genotype 


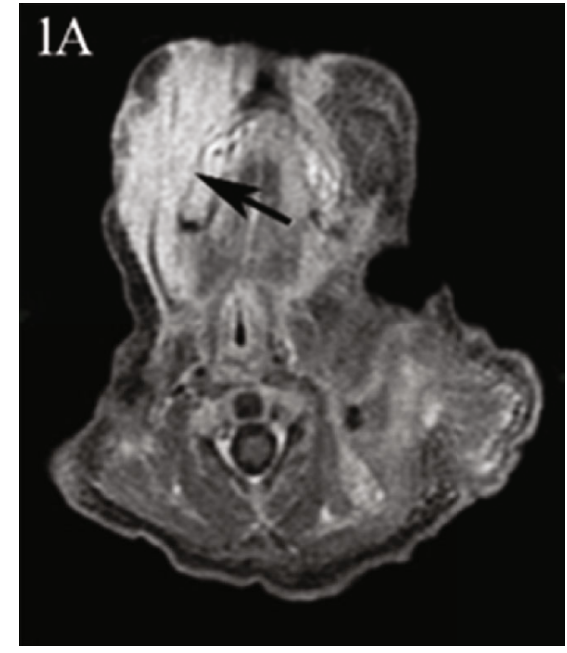

(a)

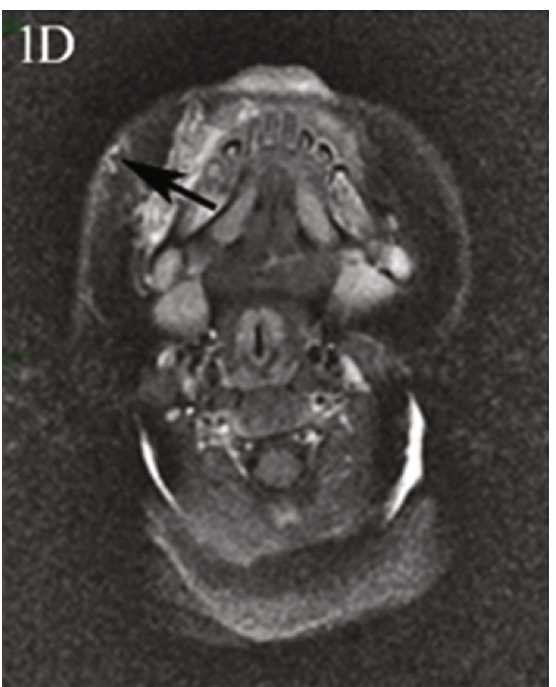

(d)

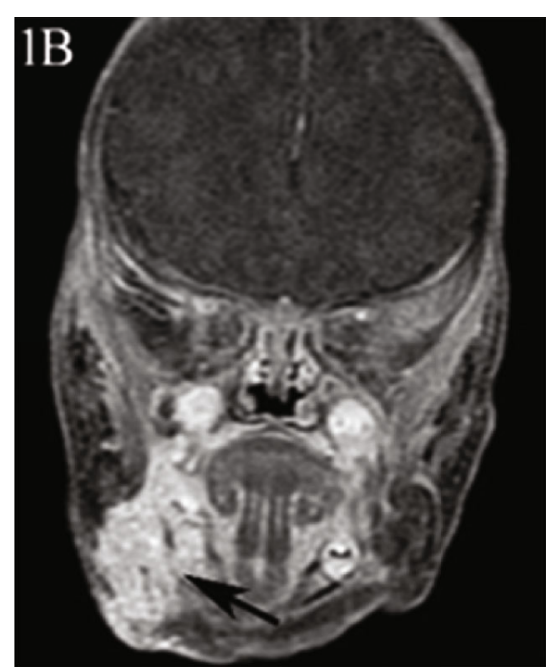

(b)

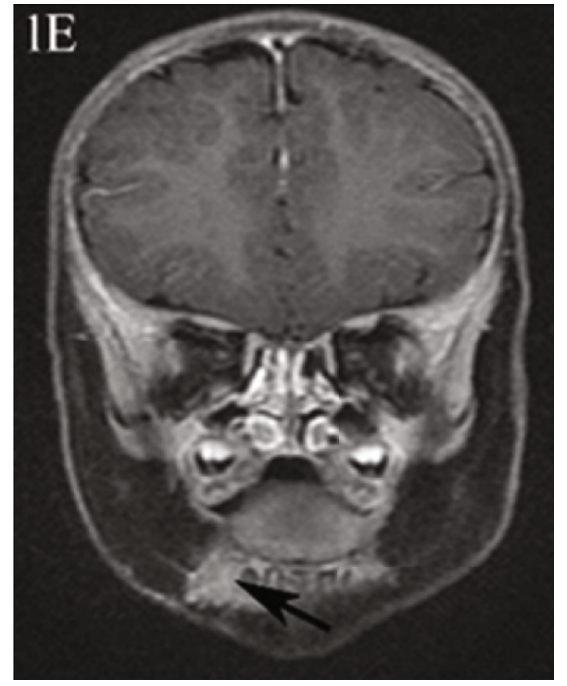

(e)

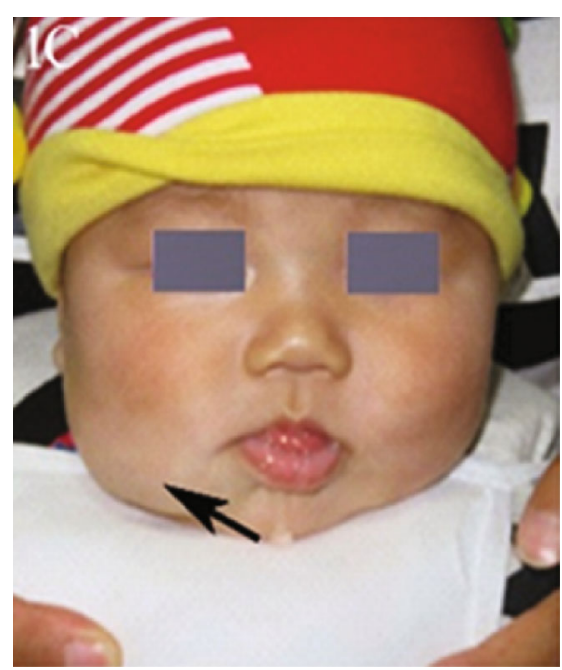

(c)

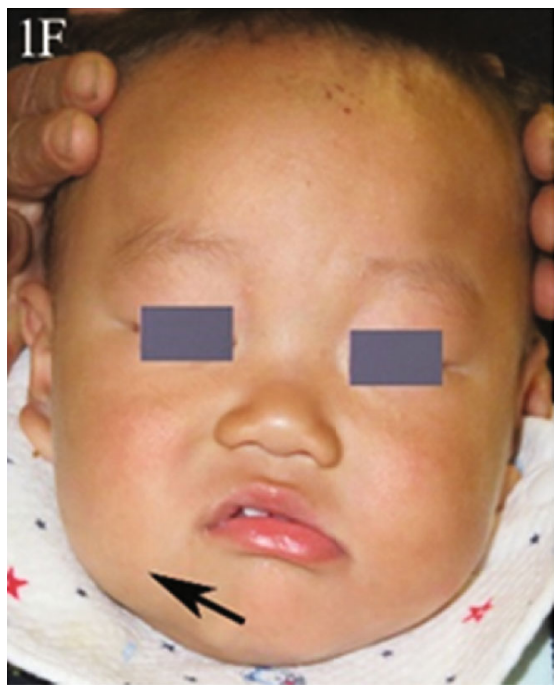

(f)

FIGURE 1: An 11-week-old boy with a deep IH on the right maxillofacial region. (a-c) Before propranolol treatment, (a-b) axial and coronal MRI $T_{1}$ WI enhancement showed obvious high signal of the right maxillofacial hemangioma. (d-f) After 8 months of propranolol treatment, (d, e) axial and coronal MRI $\mathrm{T}_{1} \mathrm{WI}$ enhancement showed only a few residual lesions.

of CYP2D6 (rs1135840) had the highest response rate, while those with the $\mathrm{C} / \mathrm{C}$ type had the lowest response rate.

3.2. Side Effects. No severe side effects including pulmonary symptoms, hypoglycemia, or bradycardia were found by physicians, parents, or guardians during the treatment. No patients needed any dose reduction of propranolol, and no one withdrew from treatment because of adverse reactions. Five children experienced slight diarrhea at the initiation of the administration of propranolol, and diarrhea disappeared in three days in most cases.

\section{Discussion}

Oral propranolol is now widely used in treating complicated IH. However, some patients do not show a good response to propranolol and need additional treatment other than pro- pranolol alone. Nowadays, there is no satisfying marker that can predict poor response to propranolol treatment.

In the present study, the impact of CYP2D6 (rs1065852 and rs1135840) gene polymorphisms on the efficacy of oral propranolol in treating IH was investigated. It showed that the patients with rs1135840 (CYP2D6) G/G homozygotes had the highest response rate, and the patients with the $\mathrm{C} / \mathrm{C}$ type had the lowest response rate after six months of treatment. The response to propranolol treatment in $\mathrm{IH}$ patients was related to gene polymorphism of rs1135840 (CYP2D6). A study investigating the relationship between polymorphisms of Cytochrome P450 2D6 and blood hydroxychloroquine levels in patients with systemic lupus erythematosus showed that patients with rs1135840 G/G homozygotes had decreased CYP2D6 activity and lower concentrations of metabolites [21]. The highest response rate in patients with rs1135840 (CYP2D6) G/G homozygote in this study might be associated with a higher serum 


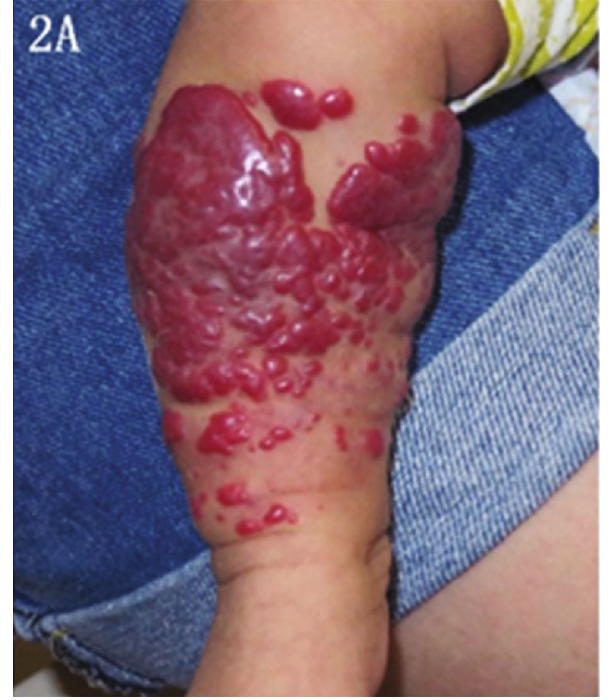

(a)

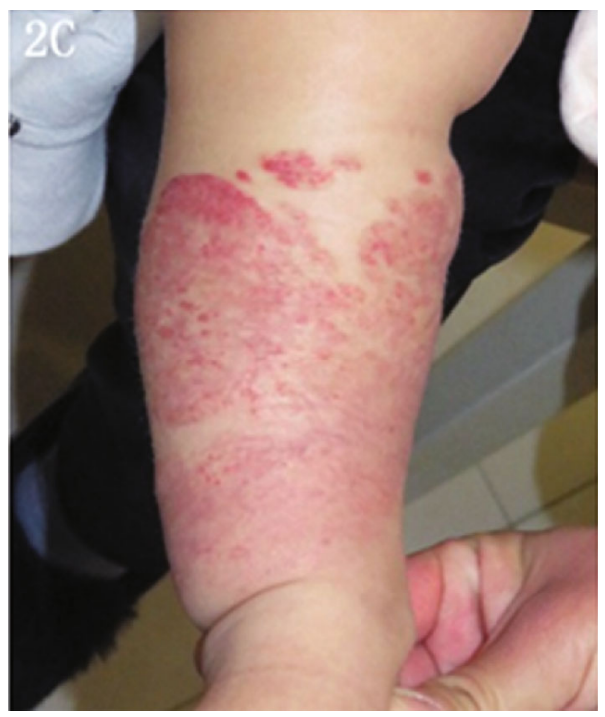

(c)

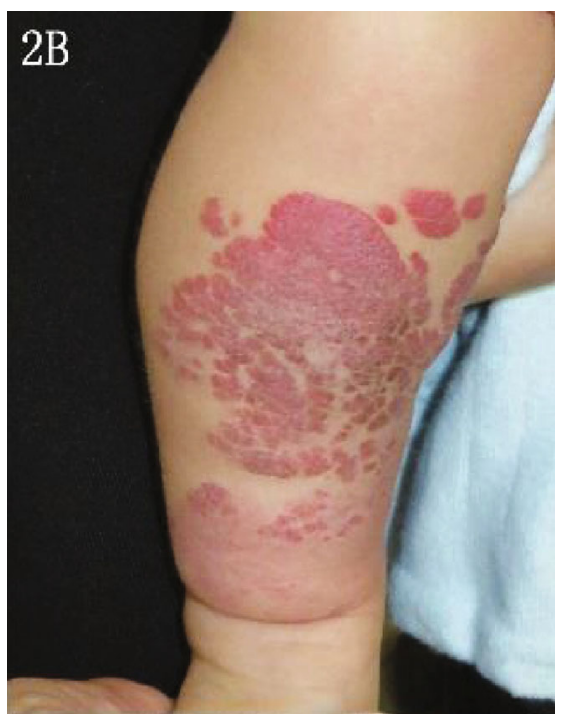

(b)

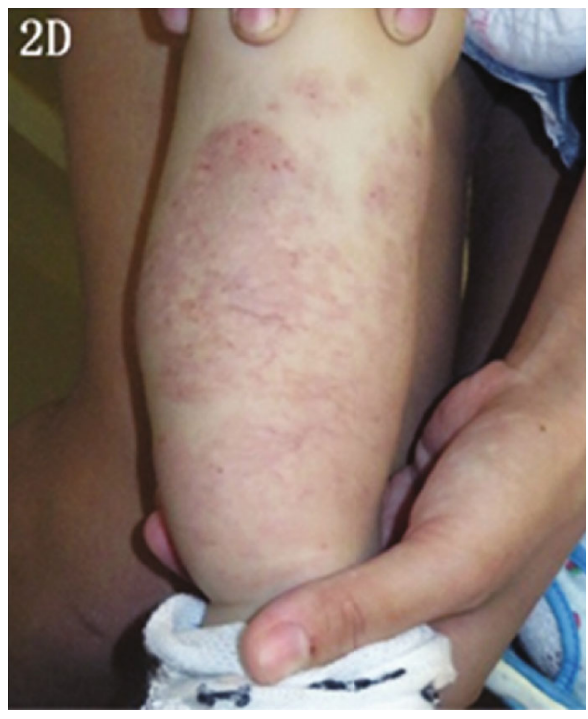

(d)

FIGURE 2: A 9-week-old girl with an IH on the right leg. (a) 1 day before propranolol treatment, (b) after 3 months of propranolol treatment, (c) after 6 months of propranolol treatment, (d) 12 months after propranolol treatment.

propranolol concentration. We speculate that for patients with rs1135840 G/C or C/C genotype, the dose of propranolol could be individualized provided the monitoring of serum propranolol concentrations. However, the speculation needs to be further investigated.

The reported propranolol target doses were diverse, and the optimal doses were inconsistent in different reports. A study showed that at the dose of $3 \mathrm{mg} \mathrm{kg}^{-1} \mathrm{day}^{-1}$, patients could get the optimal response [19]. Another study showed that no significant difference was found in the treatment response in different doses, while the side-effects of propranolol were found to be associated with a higher dose of propranolol [22]. It seems that lower propranolol dose is safer for infants with IHs. There have been studies showing the effectiveness of low-dose propranolol for IHs [23, 24], and the dose of $1.0 \mathrm{mg} \mathrm{kg}^{-1} \mathrm{day}^{-1}$ was proved to be successful in treating $\mathrm{IHs}^{24}$. In our study, 1 daily administration of pro- pranolol was chosen for three main reasons. Firstly, a study showed that the administration of propranolol once a day at a dose of $1.0 \mathrm{mg} \mathrm{kg}^{-1} \mathrm{day}^{-1}$ was effective in the treatment of superficial IH [25]. Secondly, in our previous clinical practice, we found that 1 daily administration of propranolol at the dose of $1.0 \mathrm{mg} \mathrm{kg}^{-1} \mathrm{day}^{-1}$ was also effective in treating other types of IHs including deep IHs and mixed IHs. Thirdly, there is no propranolol oral solution available in China. As the target dose of propranolol in our study was relatively low, it would be difficult for parents to divide the dose accurately to twice or three times a day. 1 daily administration could increase patient compliances. The response rate in our study after six months of treatment was satisfying. The side effects in this study were slight.

A study conducted in infants aged less than four months showed that propranolol could stop the growth of $\mathrm{IH}$, while a mild growth of IH was observed in the placebo group [26]. A 


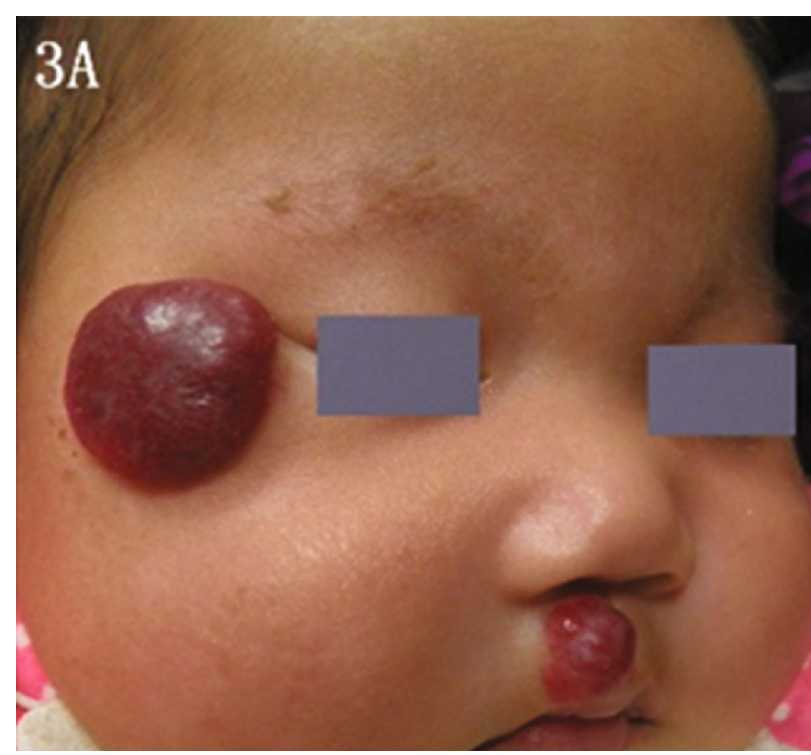

(a)

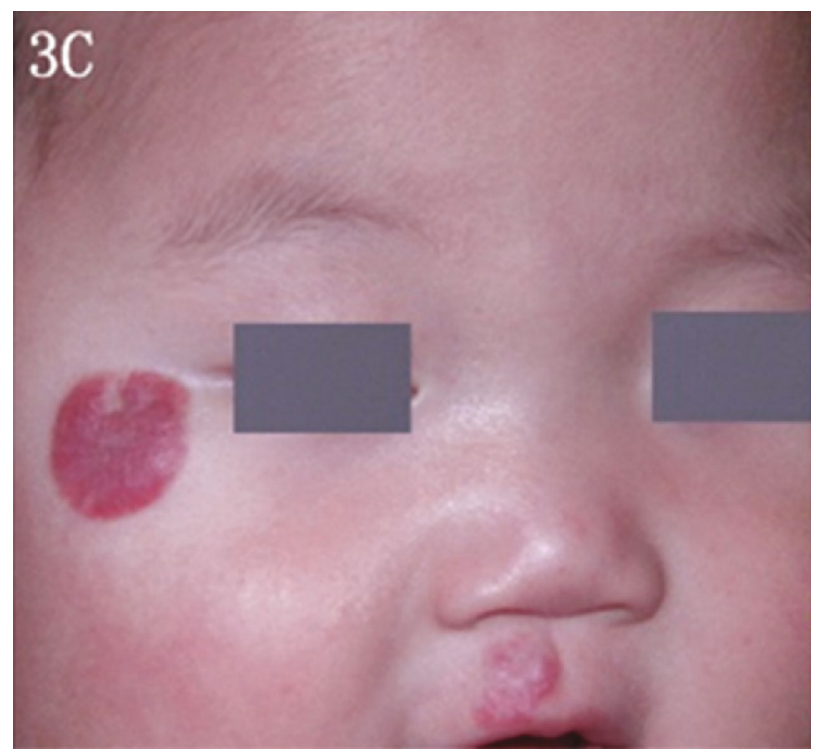

(c)

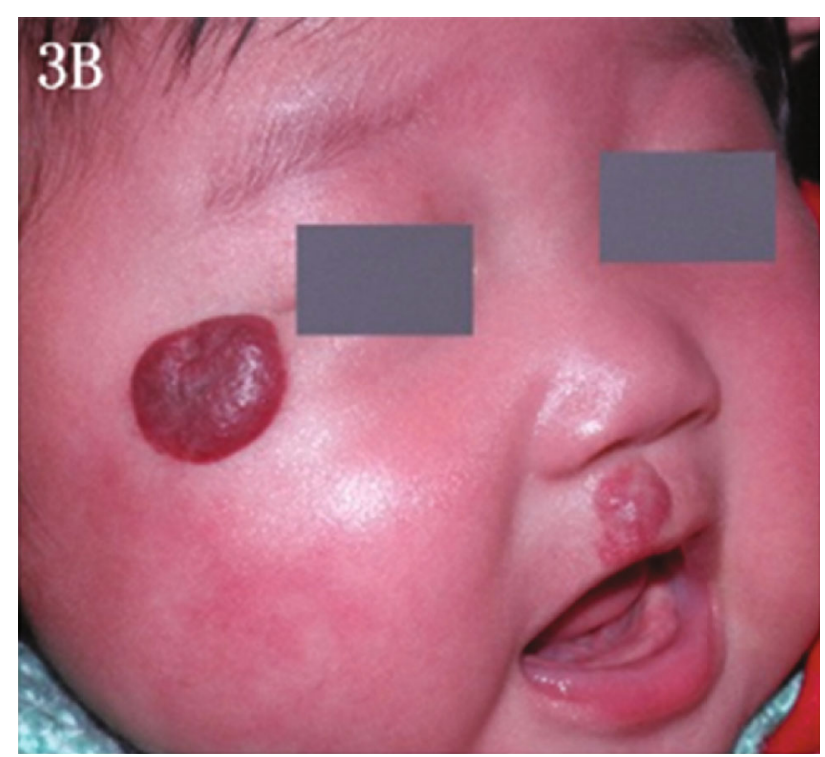

(b)

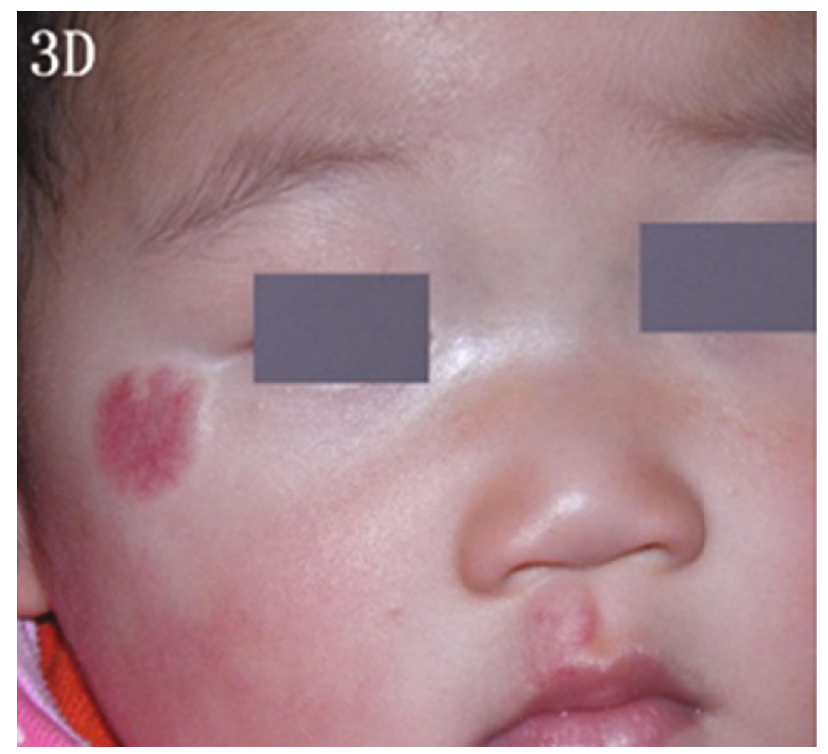

(d)

FIGURE 3: A 10-week-old girl with an IH on the right cheek. (a) 1 day before propranolol treatment, (b) after 3 months of propranolol treatment, (c) after 6 months of propranolol treatment, (d) 12 months after propranolol treatment.

study about the early growth characteristics of IHs showed that most rapid growth of $\mathrm{IH}$ appeared between 5.5 and 7.5 weeks of age [27]. For patients who need to be treated, oral propranolol is proposed to be administered as early as possible to prevent the occurrence of potential complications [28]. In our study, 52 patients were younger than 3 months old at the propranolol initiation. Most patients received the administration of propranolol about one month after the occurrence of IH. No severe side effects were noticed by physicians, parents, or guardians during the treatment. The lowdose propranolol regimen was effective and safe in young infants with $\mathrm{IH}$.

Another finding in the present study is that the changes in serum VEGF levels after one month of therapy could not be used to predict the response rate to propranolol. The exact mechanisms of propranolol in treating IH are still not clarified, but propranolol was found to be able to regulate hemangioma cell proliferation through the impact on the VEGF pathway [29]. In the present study, VEGF levels one month after the initiation of treatment decreased compared with their baseline VEGF which was consistent with a previous study [17]. However, no difference in percentage VEGF changes after one month of treatment between the poor-tomoderate responders and the good responders were found.

There were a few limitations in the present study. Firstly, the study included a small number of patients. The findings need to be proved in studies with a larger number of patients. Secondly, there was no placebo group in this study. Though the study was a prospective one, no patients' guardians were willing to take a placebo during the treatment. 
TABle 2: Associations between CYP2D6 genotypes and the treatment responses.

\begin{tabular}{lcccc}
\hline & $N$ & $\begin{array}{c}\text { Good } \\
\text { responders }\end{array}$ & $\begin{array}{c}\text { Poor-to-moderate } \\
\text { responders }\end{array}$ & $p$ \\
\hline rs1065852 & & & & 0.541 \\
G/G $n(\%)$ & 17 & $11(64.7)$ & $6(35.3)$ & \\
A/G $n(\%)$ & 39 & $30(76.9)$ & $9(23.1)$ & \\
A/A $n(\%)$ & 16 & $13(81.3)$ & $3(18.8)$ & \\
rs1135840 & & & & 0.031 \\
$\mathrm{C} / \mathrm{C} n(\%)$ & 11 & $6(54.5)$ & $5(45.5)$ & \\
$\mathrm{C} / \mathrm{G} n(\%)$ & 31 & $21(67.7)$ & $10(32.3)$ & \\
$\mathrm{G} / \mathrm{G} n(\%)$ & 30 & $27(90.0)$ & $3(10.0)$ & \\
\hline
\end{tabular}

Categorical data were reported as proportions ( $n$ with \%)and compared using chi-square test.

\section{Conclusion}

The response to propranolol treatment in IH patients was associated with the gene polymorphism of CYP2D6 (rs1135840). A low-dose propranolol regimen was effective and safe in young infants with $\mathrm{IH}$. The change of serum VEGF levels after one month's treatment could not be used to predict the response rate to propranolol.

\section{Data Availability}

The data used to support the findings of this study are available from the corresponding author upon request.

\section{Conflicts of Interest}

The authors declare that they have no conflicts of interest.

\section{Authors' Contributions}

WLD, XQ, and WY designed the study. ZK recorded clinical data and evaluated clinical efficacy. WLD and XQ collected samples and wrote the manuscript. XQ and LXL performed the experiments. All authors have read and approved the manuscript. Lidan Wang and Kai Zheng contributed equally to this work.

\section{Acknowledgments}

We thank Peiwei Zhao (precision medicine lab, Wuhan Children's Hospital) for his technical support. The study was supported by Wuhan Municipal Health Commission scientific research project (grant agreement number: WX17D18). No authors have other financial interests that could create a conflict of interest with regard to the work.

\section{References}

[1] M. Novoa, E. Baselga, S. Beltran et al., "Interventions for infantile haemangiomas of the skin," Cochrane Database of Systematic Reviews, vol. 4, article CD006545, 2018.

[2] A. N. Haggstrom, B. A. Drolet, E. Baselga et al., "Prospective study of infantile hemangiomas: clinical characteristics pre- dicting complications and treatment," Pediatrics, vol. 118, no. 3, pp. 882-887, 2006.

[3] Y. Lou, W. J. Peng, Y. Cao, D. S. Cao, J. Xie, and H. H. Li, “The effectiveness of propranolol in treating infantile haemangiomas: a meta-analysis including 35 studies," British Journal of Clinical Pharmacology, vol. 78, no. 1, pp. 44-57, 2014.

[4] R. J. Phillips, Z. Lokmic, C. M. Crock, and A. Penington, "Infantile haemangiomas that failed treatment with propranolol: clinical and histopathological features," Journal of Paediatrics and Child Health, vol. 50, no. 8, pp. 619-625, 2014.

[5] S. Caussé, H. Aubert, M. Saint-Jean et al., "Propranolol-resistant infantile haemangiomas," The British Journal of Dermatology, vol. 169, no. 1, pp. 125-129, 2013.

[6] J. C. Lopez-Gutierrez, "Clinical and economic impact of surgery for treating infantile hemangiomas in the era of propranolol: overview of single-center experience from $\mathrm{La} \mathrm{Paz}$ Hospital, Madrid," European Journal of Pediatrics, vol. 178, no. 1, pp. 1-6, 2019.

[7] D. H. Darrow, A. K. Greene, A. J. Mancini, A. J. Nopper, and Section on Dermatology, Section on Otolaryngology-Head and Neck Surgery, and Section on Plastic Surgery, "Diagnosis and management of infantile hemangioma," Pediatrics, vol. 136, no. 4, pp. e1060-e1104, 2015.

[8] V. Pandey, P. Tiwari, S. P. Sharma, R. Kumar, and O. P. Singh, "Role of intralesional bleomycin and intralesional triamcinolone therapy in residual haemangioma following propranolol," International Journal of Oral and Maxillofacial Surgery, vol. 47, no. 7, pp. 908-912, 2018.

[9] C. Leaute-Labreze, S. Prey, and K. Ezzedine, "Infantile haemangioma: part I. pathophysiology, epidemiology, clinical features, life cycle and associated structural abnormalities," Journal of the European Academy of Dermatology and Venereology, vol. 25, no. 11, pp. 1245-1253, 2011.

[10] J. H. Chong, S. Prey, H. T. Mya, A. Delarue, and C. Labreze, "Can the extent of heart rate reduction predict the clinical response of infantile haemangiomas to propranolol?," The British Journal of Dermatology, vol. 178, no. 3, pp. e196e197, 2018.

[11] H. H. Zhou, L. B. Anthony, D. M. Roden, and A. J. Wood, "Quinidine reduces clearance of (+)-propranolol more than (-)-propranolol through marked reduction in 4-hydroxylation," Clinical Pharmacology and Therapeutics, vol. 47, no. 6, pp. 686-693, 1990.

[12] I. Johansson, M. Oscarson, Q. Y. Yue, L. Bertilsson, F. Sjoqvist, and M. Ingelman-Sundberg, "Genetic analysis of the Chinese cytochrome P4502D locus: characterization of variant CYP2D6 genes present in subjects with diminished capacity for debrisoquine hydroxylation," Molecular Pharmacology, vol. 46, no. 3, pp. 452-459, 1994.

[13] T. Fukuda, I. Yamamoto, Y. Nishida et al., "Effect of the CYP2D6*10 genotype on venlafaxine pharmacokinetics in healthy adult volunteers," British Journal of Clinical Pharmacology, vol. 47, no. 4, pp. 450-453, 1999.

[14] M. L. Lai, S. L. Wang, M. D. Lai, E. T. Lin, M. Tse, and J. D. Huang, "Propranolol disposition in Chinese subjects of different CYP2D6 genotypes," Clinical Pharmacology and Therapeutics, vol. 58, no. 3, pp. 264-268, 1995.

[15] C. H. Storch and P. H. Hoeger, "Propranolol for infantile haemangiomas: insights into the molecular mechanisms of action," The British Journal of Dermatology, vol. 163, no. 2, pp. 269-274, 2010. 
[16] L. Zhu, J. Xie, Z. Liu et al., "Pigment epithelium-derived factor/vascular endothelial growth factor ratio plays a crucial role in the spontaneous regression of infant hemangioma and in the therapeutic effect of propranolol," Cancer Science, vol. 109, no. 6, pp. 1981-1994, 2018.

[17] X. D. Chen, G. Ma, J. L. Huang et al., "Serum-level changes of vascular endothelial growth factor in children with infantile hemangioma after oral propranolol therapy," Pediatric Dermatology, vol. 30, no. 5, pp. 549-553, 2013.

[18] L. Babiak-Choroszczak, K. Giżewska-Kacprzak, E. Gawrych et al., "Serum concentrations of VEGF and bFGF in the course of propranolol therapy of infantile hemangioma in children: are we closer to understand the mechanism of action of propranolol on hemangiomas?," Advances in Clinical and Experimental Medicine, vol. 27, no. 5, pp. 703-710, 2018.

[19] C. Léauté-Labrèze, P. Hoeger, J. Mazereeuw-Hautier et al., "A randomized, controlled trial of oral propranolol in infantile hemangioma," The New England Journal of Medicine, vol. 372, no. 8, pp. 735-746, 2015.

[20] K. G. Chiller, D. Passaro, and I. J. Frieden, "Hemangiomas of infancy: clinical characteristics, morphologic subtypes, and their relationship to race, ethnicity, and sex," Archives of Dermatology, vol. 138, no. 12, pp. 1567-1576, 2002.

[21] J. Y. Lee, N. Vinayagamoorthy, K. Han et al., “Association of polymorphisms of cytochrome P450 2D6 with blood hydroxychloroquine levels in patients with systemic lupus erythematosus," Arthritis \& Rhematology, vol. 68, no. 1, pp. 184-190, 2016.

[22] E. Wedgeworth, M. Glover, A. D. Irvine et al., "Propranolol in the treatment of infantile haemangiomas: lessons from the European propranolol in the treatment of complicated Haemangiomas (PITCH) taskforce survey," The British Journal of Dermatology, vol. 174, no. 3, pp. 594-601, 2016.

[23] C. E. Tan, T. Itinteang, P. Leadbitter, R. Marsh, and S. T. Tan, "Low-dose propranolol regimen for infantile haemangioma," Journal of Paediatrics and Child Health, vol. 51, no. 4, pp. 419-424, 2015.

[24] Y. Duzenli Kar, Z. C. Ozdemir, B. Acu, and O. Bor, "Infantile hemangioma: efficacy of low-dose propranolol and of intralesional bleomycin injection for propranolol non-response," Pediatrics International, vol. 61, no. 5, pp. 459-464, 2019.

[25] H. Gong, D. P. Xu, Y. X. Li, C. Cheng, G. Li, and X. K. Wang, "Evaluation of the efficacy and safety of propranolol, timolol maleate, and the combination of the two, in the treatment of superficial infantile haemangiomas," The British Journal of Oral \& Maxillofacial Surgery, vol. 53, no. 9, pp. 836-840, 2015.

[26] C. Léauté-Labrèze, E. Dumas de la Roque, F. Nacka et al., "Double-blind randomized pilot trial evaluating the efficacy of oral propranolol on infantile haemangiomas in infants $<4$ months of age," The British Journal of Dermatology, vol. 169, no. 1, pp. 181-183, 2013.

[27] M. M. Tollefson and I. J. Frieden, "Early growth of infantile hemangiomas: what parents' photographs tell us," Pediatrics, vol. 130, no. 2, pp. e314-e320, 2012.

[28] C. Leaute-Labreze, J. I. Harper, and P. H. Hoeger, "Infantile haemangioma," The Lancet, vol. 390, no. 10089, pp. 85-94, 2017.

[29] Y. Ji, S. Chen, C. Xu, L. Li, and B. Xiang, "The use of propranolol in the treatment of infantile haemangiomas: an update on potential mechanisms of action," The British Journal of Dermatology, vol. 172, no. 1, pp. 24-32, 2015. 